\begin{tabular}{|c|c|c|c|}
\hline Eiszeitalter $u$. Gegenwart & 40 & $111-119$ & Hannover 1990 \\
\hline
\end{tabular}

\title{
Spätglazial und Holozän auf der Stolzenauer Terrasse im Mittelwesertal
}

\author{
Susanne Lipps \& Gerfried Caspers *)
}

\begin{abstract}
Holocene, fluvial features, braided streams, paleomeanders, floodplains, dunes,
lithostratigraphy, alluvial soils, loam, sand, size distribution, marker bed, Laacher See Tuff, paleorelief
\end{abstract}

Lower Saxony, Northwestern German Plain, Weser River, TK 3420

\begin{abstract}
Kurzfassung: 1. Der Umschwung vom "braided river" zum mäandrierenden Fluß hat an der Mittelweser bei Stolzenau spätestens während des Alleröds, vielleicht schon während des Böllings stattgefunden. Im Alleröd kam es bereits zur Ablagerung eines Hochflut-/ Auelehms durch einen mäandrierenden Fluß. Die großen, mäanderförmigen Paläorinnen auf der Stolzenauer Terrasse sind durchweg im Spätglazial entstanden. Das höhere Niveau der Stolzenauer Terrasse stellt ein Übergangsstadium zwischen der eigentlichen Niederterrasse (durch einen "braided river" aufgeschüttet) und der spätglazialen Stolzenauer Terrasse dar. Das höhere Niveau ist ebenfalls mindestens im Spätglazial entstanden.
\end{abstract}

2. Bis ins Präboreal hinein kam es in den Rinnen der Stolzenauer Terrasse zur Bildung von Mudden und Torfen. Der Anteil organischer Substanz in den Mudden ist verhältnismäßig hoch. Unterhalb einer Entkalkungstiefe von $2 \mathrm{~m}$ enthalten die Mudden um $5 \%$ Kalk. Auf den Flächen kam es zu Hochflut-/ Auelehmablagerung. Diese ist auf die nähere Umgebung der Paläorinnen begrenzt.

3. Boreal und älteres Atlantikum sind Zeiten relativer Ruhe. Aus diesen Zeiten sind nur wenige Sedimente überliefert, auch die Torfbildung war gering. Im ausgehenden Atlantikum kam es dann zu einer Erosionsphase, in deren Verlauf sich die Weser um etwa $3 \mathrm{~m}$ in die Stolzenauer Terrasse eintiefte; die heutige Weseraue entstand.

4. Auf der Stolzenauer Terrasse wurden in den Paläorinnen vom jüngeren Atlantikum bis in die vorrömische Eisenzeit hinein Torfe und Mudden mit hohem Anteil organischer Substanz abgesetzt. Mit der Bronzezeit setzte dann in der Weseraue die Auelehm-Sedimentation ein. Um die Zeitwende herum trat wieder eine Ruhephase ein, und es kam zu einer weiteren, geringfügigen Erosion.

5. Mit dem Beginn des Mittelalters setzte eine starke Sedimentation sowohl in der Aue als auch auf der Stolzenauer Terrasse ein. Während des Mittelalters und teilweise noch in der Neuzeit wurden wesentlich mehr feinklastische Sedi-

*) Anschrift der Verfasser: G. CASPERS, Niedersächsisches Landesamt für Bodenforschung, Stilleweg 2, 3000 Hannover 51. Dr. S. Lipps, Mozartstr. 7, D -4000 Düsseldorf 1. mente abgesetzt als in den vorangegangenen Abschnitten des Spätglazials und Holozäns. Der organische Anteil in den Mudden ist gering. Torfbildung ist selten. In den Paläorinnen der Stolzenauer Terrasse kam es, wie auch schon im Subboreal, zu Erosionserscheinungen mit nachfolgender Muddesedimentation. Eine kontinuierliche Auelehmsedimentation fand nur in der Talaue statt. Auf der Stolzenauer Terrasse kam es nur im ausgehenden Mittelalter, bedingt durch außerordentliche Hochwässer, zu einer Auelehmsedimentation in der näheren Umgebung der Rinnen.

\section{[Late Glacial and Holocene on the Stolzenau Terrace in the Middle Weser Valley]}

Abstract: 1. At the middle Weser the change from a braided to a meandering river took place at the latest during the Alleröd, possibly already during the Bölling. During the Alleröd high-flood loam was deposited by a meandering river. The large paleomeanders on the Stolzenau Terrace developed during the Late Glacial. A higher level of the Stolzenau Terrace can be distinguished. It seems to be a transitional terrace between the "Niederterrasse" (accumulated by a braided river) and the Stolzenau Terrace. The higher level has been formed during the Late Glacial or even earlier.

2. Up to the Preboreal formation of gyttjas and peat took place in the channels of the Stolzenau Terrace. The gyttjas contain a relatively high amount of organic material. Below a decalcification depth of $2 \mathrm{~m}$ the content of calcium carbonate reaches about $5 \%$. On the flood plains sedimentation of high-flood loam takes place which is limited to the nearer surroundings of the fossil river channels.

3. Boreal and older Atlantic are times of rest. Only few sediments from those times can be found today. The peat formation is negligible. In the younger Atlantic an erosion phase occurs, during which the Weser incises about $3 \mathrm{~m}$ into the Stolzenau Terrace. The recent valley floor is formed.

4. In the channnels of the Stolzenau Terrace peat and gyttjas with high contents of organic substance are formed from the younger Atlantic up to the preroman iron age. With the bronze age deposition of high-flood loam sets in 
on the recent valley floor. At the beginning of the Christian era this sedimentation ceases, and another, minor incision takes place.

5. With the beginning of the middle Ages a strong sedimentation in the recent valley floor as well as on the Stolzenau Terrace sets in. From then on up to modern times much more finegrained sediments are deposited than during the earlier parts of the Late Glacial and Holocene. The content of organic material in gyttjas is minimal, peat is scarcely formed. In the fossil river channels of the Stolzenau Terrace erosion with following gyttja formation takes place again. A continuous high-flood loam sedimentation exclusively occurs on the recent valley floor. On the Stolzenau Terrace highflood loam deposition probably only takes place during the late middle Ages. Limited to the nearer surroundings of the channels it is due to extraordinary floods.

\section{Einleitung}

Das Arbeitsgebiet erstreckt sich im oberen Mittelwesertal am Westrand der Talaue zwischen Stolzenau und Liebenau (vgl. Abb. 1). Im Übergangsbereich zur Niederterrasse liegen hier zahlreiche verlassene Rinnen außerhalb des eigentlichen Überflutungsbereiches der Weser (Stolzenauer Terrasse). Im Bereich der heute noch regelmäßig überfluteten Talaue konnten bisher keine älteren als jungbronze- bis eisenzeitliche Auelehme sowie spätatlantische Rinnensedimente (Mudden, Torfe) gefunden werden (vgl. LIPPS 1987, 1988). Ein Auelehm, dessen Bildung LÜTTIG \& MEYER (1980) im Atlantikum vermuteten, konnte jetzt zweifelsfrei zum qh (1) (ältester holozäner Auelehm) gestellt werden und ist somit eisenzeitlich.

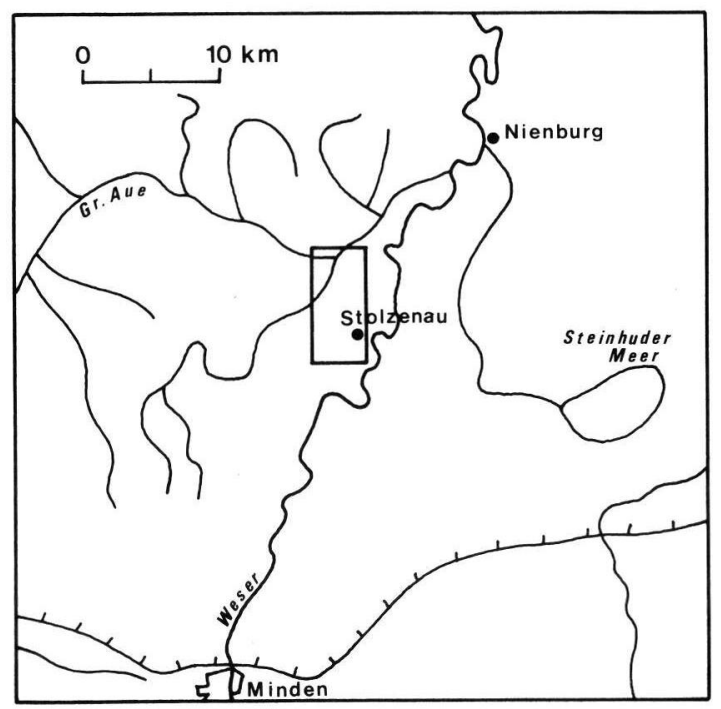

Abb. 1: Übersichtskarte.
Spätglaziale bis atlantische Sedimente wurden im Bereich der verlassenen Rinnen am Westrand der Aue bei Stolzenau vermutet. Diese Rinnen liegen $2-5 \mathrm{~m}$ über den Rinnen der heutigen Talaue und besitzen größtenteils die Form großer, gut ausgebildeter Mäander. Im Mittelwesertal treten solche Rinnen noch vereinzelt in anderen Gebieten auf. Jedoch zeichnet sich das Vorkommen westlich von Stolzenau durch seinen außerordentlich guten Erhaltungszustand aus, der wohl dem Umstand zu verdanken ist, daß die Weser hier im Verlauf des Holozäns ihren Lauf weit nach Osten verlagert hat.

Die Stolzenauer Paläomäander ähneln in ihren Merkmalen (Größe, Form, Höhenlage über den rezenten Mäandern) stark den von KOZARSKI (1983) aus dem polnischen Tiefland beschriebenen, ins Spätglazial datierten Mäandern der älteren Generation. Auch in anderen Flußgebieten Mitteleuropas treten vergleichbare Mäander auf, deren Ursprung im Spätglazial oder älteren Holozän zu suchen ist, so am Main (SCHIRMER 1980, 1983) und an der Ems (MÜLLER 1956, ROESCHMANN 1960).

Im Rahmen eines von der DFG geförderten Forschungsprojekts unter der Leitung von Dr. C. HINZE und Dr. H. JORDAN entstand am Niedersächsischen Landesamt für Bodenforschung (NLfB) die vorliegende Arbeit (ausführliche Darstellung in unveröff. Berichten LIPPS 1989 und CASPERS 1989).

Mit Hilfe von 117 Handbohrungen (Teufen bis zu $7 \mathrm{~m}$ ) sowie Aufnahme von Aufschlüssen und der Auswertung von Bohrungen aus dem Vorlaufprogramm konnte die Verbreitung spätglazialer und früh- bis mittelholozäner Sedimente im oberen Mittelwesertal recht genau ermittelt werden. Pollenanalytische und ${ }^{14} \mathrm{C}$-Datierungen ermöglichen es, das Sedimentationsgeschehen nachzuvollziehen. Die Datierung von 4 Holzproben mittels der ${ }^{14} \mathrm{C}$-Analyse übernahm dankenswerterweise Herr Dr. M. A. GEYH (NLfB). Außerdem wurde an 42 Sedimentproben Korngrößenverteilung, Kalkgehalt und Glühverlust bestimmt (Sedimentlabor des NLfB (Dr. MATTIAT) sowie mikrobotanisches Labor der BGR (Dr. H. MÜLLER)). Allen, die an den Untersuchungen beteiligt waren, gilt unser Dank. Herr Dr. RiezeBos vom Physikalischen und Bodenkundlichen Labor der Universität Amsterdam übernahm dankenswerterweise die Untersuchung pyroklastischen Materials.

Hilfreiche wissenschaftliche Diskussionen zum Thema wurden mit zahlreichen Mitarbeitern des NLfB und anderer Stellen, die hier nicht alle namentlich aufgeführt werden können, geführt. Ihnen allen gilt unser Dank. Schließlich bedanken wir uns bei all jenen, die durch ihren unermüdlichen Einsatz im Gelände zum erfolgreichen Abschluß der Arbeiten beigetragen haben. 


\section{Morphologie der Stolzenauer Terrasse}

Im Gegensatz zu der eigentlichen Talaue, deren Morphologie durch eine geschlossene Auelehmdecke weitgehend verdeckt wird, weist die Stolzenauer Terrasse eine lebhafte Morphologie auf (vgl. Abb. 2). Die Abgrenzung der Talaue gegenüber der Stolzenauer Terrasse ist durch eine deutliche Kante gegeben. Die Kante wird durch eine Reihe von Mäanderaußenbögen gebildet, setzt sich also aus ehemaligen oder auch rezenten Prallhängen der Weser zusammen, wobei die vorspringenden Spitzen wohl durch Hochwässer geglättet wurden. Die Verbindung aller derartiger Mäanderaußenbögen wird von SCHIRMER (1983) als Nahtrinne bezeichnet.

Die eigentliche Stolzenauer Terrasse ist ebenfalls durch eine, wenn auch nicht immer eindeutige Kante von einem höheren Niveau getrennt, dessen Morphologie bedeutende Abweichungen gegenüber den Oberflächenformen der Stolzenauer Terrasse aufweist. Auch hier wird die Kante durch Mäanderaußenbögen gebildet, sie ist aber durch Hochwasser nicht wesentlich geglättet worden, ein Indiz für das seltene Auftreten von flächenhaften Hochwässern auf der Stolzenauer Terrasse.

Bei dem höheren Niveau der Stolzenauer Terrasse scheint es sich um ein Übergangsstadium zwischen der Niederterrasse, die von einem "braided river" aufgeschüttet wurde, und der Stolzenauer Terrasse zu handeln, die eindeutig von einem mäandrierenden Fluß geprägt wurde. Auf dem höheren Niveau sind sowohl Reste eines verwilderten Flußsystems mit schmalen, oft muldenförmigen, mehr oder weniger gradlinig verlaufenden Rinnen zu erkennen als auch unvollkommen ausgebildete, kleine Paläomäander mit muldenförmigem oder auch schon ansatzweise kastenförmigem Querschnitt. Die kleinen Mäander finden sich gehäuft in der Nähe der Kante, die zur Stolzenauer Terrasse überleitet. Der Übergang zur eigentlichen Niederterrasse kann nicht genau festgelegt werden. Es gibt sowohl Bereiche, in denen die Niederterrasse von vielen Rinnen durchzogen ist, die zum Teil heute von kleineren Wasserläufen benutzt werden, als auch Bereiche, in denen keine Rinnen zu erkennen sind und die fast eben sind.

Verschiedene morphologische Elemente sind typisch für die Stolzenauer Terrasse. Die großen Mäanderbögen sind durch kastentalförmige Rinnen gekennzeichnet, die etwa $200 \mathrm{~m}$ Breite erreichen. Die Kante zum Prallhang hin ist dabei durchweg deutlich ausgeprägt und etwa $2 \mathrm{~m}$ hoch, wohingegen die Kante am Gleithang wesentlich flacher und weniger deutlich ausgebildet ist. Auch sie erreicht aber in der Regel noch einige dm Höhe, und der Kanteneindruck wird oft noch durch die Bodennutzung verstärkt. Während in der Rinne Grünland zu finden ist, wird der Gleithang normalerweise ackerbaulich genutzt. In den Rinnen des höheren Niveaus ist größtenteils der Muldentaltypus vertreten, wenn sich auch vereinzelt bei den Übergangstypen die Kastentalform andeutet. Aber auch auf der eigentlichen Stolzenauer Terrasse gibt es kleine Muldentälchen. Der Innenbogen eines Paläomäanders umschließt den höhergelegenen Mäanderkern, der sich zum Mäander hin als Gleithang relativ ungegliedert abdacht. Zur anderen Seite hin wird jeder Mäander durch ein jüngeres Stadium (also einen jüngeren Prallhang) abgeschnitten. Das dort herrschende, starke Gefälle vom Mäanderkern zur jüngeren Rinne hin trägt zur Entstehung von muldenartigen Erosionsformen bei, die ihren Ausgangspunkt im zentralen Teil des Mäanderkerns haben und in die jüngere Rinne münden.

Die großen Rinnen der Mäanderbögen sind in ihrem zentralen Teil mit Altarmsedimenten und Niedermoortorfen verfüllt. Anders sieht es jedoch in den Bereichen aus, die an jüngere Rinnen angrenzen, beziehungsweise von diesen seitlich angeschnitten wurden. Am (in Fließrichtung) Beginn der Mäanderschleife ist die Rinne stets durch einen Schwemmfächer verschüttet (vgl. Abb. 2), der aus sandigem Material aufgebaut wird. Der meist zungenförmige Schwemmfächer ist in der Regel eindeutig anhand der ackerbaulichen oder forstlichen Nutzung vom tieferliegenden, zentralen Rinnenbereich abzugrenzen. Die Genese eines solchen Schwemmfächers hat man sich wohl so vorzustellen, daß die ältere, vom Fluß schon verlassene Rinne bei Hochwasser erneut benutzt wird, wobei das Wasser aus den flußaufwärts gelegenen Bereichen von der benachbarten jüngeren Rinne her zutritt. Dabei tritt schlagartig eine Verminderung der Fließgeschwindigkeit ein, die die Ablagerung von sandigem Material im oberen Bereich des Altarms zur Folge hat. Die ältere Rinne wird mehr und mehr zugeschüttet und dadurch immer seltener benutzt. Im zentralen Bereich kommt es zur Ablagerung von Stillwassersedimenten und später zur Torfbildung.

Ähnlich muß man sich die Entstehung der Sandbarren vorstellen, die die älteren Rinnen am flußabwärtigen Ende gegenüber den jeweils jüngeren verschließen. Auch sie fallen sofort durch ihre ackerbauliche Nutzung auf. Sie nehmen aber deutlich weniger Raum ein als die Schwemmfächer, und sie besitzen auch keine Fächerform. Ihre Form ist mit der eines Trapezes zu vergleichen. Auch sie dürften durch Verschüttung von den jüngeren Rinnen aus entstanden sein, wobei allerdings das Material (entgegen der eigentlichen Fließrichtung) nicht allzuweit transportiert werden konnte. 

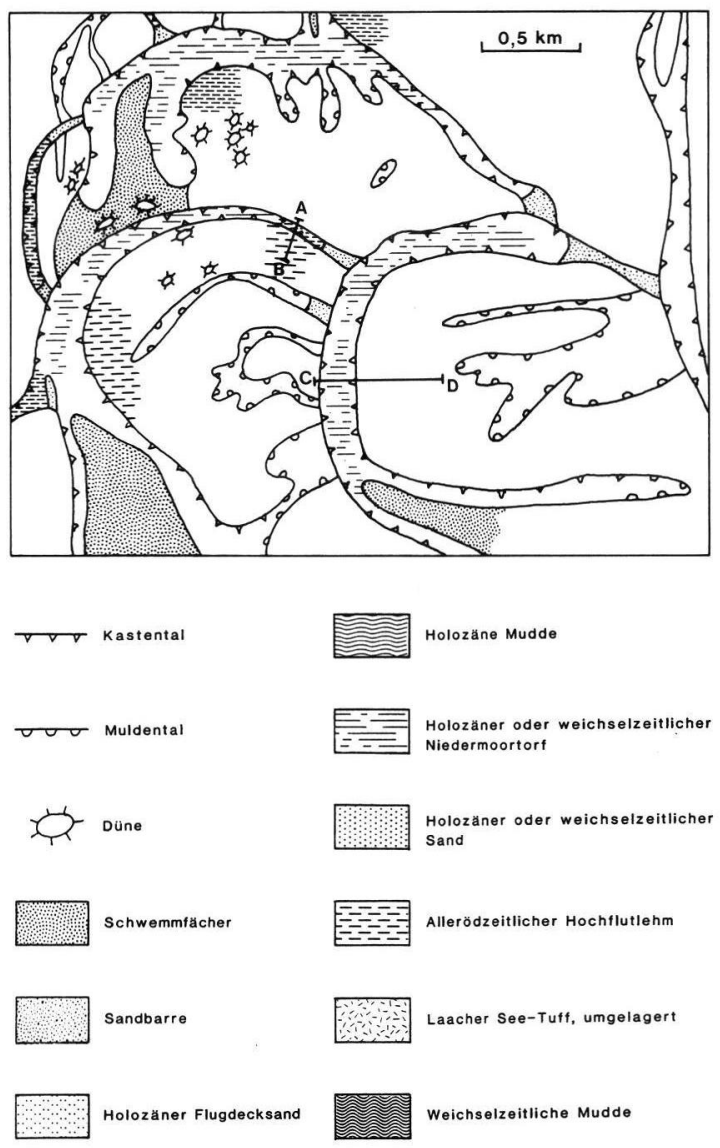

Holozäner Flugdecksand

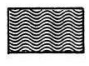

Weichselzeitliche Mudde

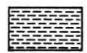

Mittelalterlicher Auelehm

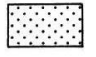

Weichselzeitliche, kiesige Sandmudde

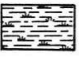

Holozäner Niedermoortorf

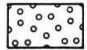

Niederterrassensand und $-k i e s$

Abb. 2: Paläomäander auf der Stolzenauer Terrasse.

Eine geschlossene Hochflut-/ Auelehmdecke fehlt der Stolzenauer Terrasse. Nur in unmittelbarer Umgebung der Rinnen, bevorzugt auf den tiefergelegenen Gleithängen, findet man eine mehrere $\mathrm{dm}$ bis $2 \mathrm{~m}$ mächtige Hochflut-/Auelehmdecke vor. Die Verbreitung der Lehmdecken ist lückenhaft. Sie greifen etwa 200 bis $500 \mathrm{~m}$ weit auf den Mäanderkern aus. Am Prallhang findet man sie nur vereinzelt. Hier können sie auch auf das höhere Niveau der Terrasse ausgreifen, während sie im allgemeinen auf die Stolzenauer Terrasse beschränkt sind. Anhand der Hochflut-/Auelehmverbereitung wird offensichtlich, daß die Stolzenauer Terrasse zur Zeit des mäandrierenden Flusses relativ selten überflutet wurde.

Die zentralen, hochgelegenen Bereiche der Mäanderkerne besitzen keine Lehmdecke. In der Regel tritt hier Wesersand an die Oberfläche, der bereits in $1-2 \mathrm{~m}$ Tiefe in Kiese übergeht. Die Aufschlußsituation in diesen Kiesen und Sanden ist äußerst ungünstig. Wahrscheinlich handelt es sich jedoch im oberen Bereich um Ablagerungen eines mäandrierenden Flusses (mdl. Mitt. von KARSTEN MEINKE, NLfB), so $\mathrm{da} ß$ anzunehmen ist, daß nicht nur die Rinnen, sondern auch die Mäanderkerne nicht der Niederterrasse (die von einem "braided river" aufgeschüttet wurde), sondern einer zeitlich eigenständigen, wahrscheinlich im Spätglazial anzusiedelnden Bildung, eben der Stolzenauer Terrasse, angehören.

Als bemerkenswerte Formen fallen auf der Stolzenauer Terrasse noch zahlreiche kleine Dünen auf. Sie sind sowohl auf der eigentlichen Terrasse als auch auf dem höheren Niveau zu finden. Sie treten oft in Schwärmen auf und sind gelegentlich mit dünnen Flugsanddecken vergesellschaftet. Sogar innerhalb der Rinnen sind sie auf Schwemmfächern zu finden. Vermutlich handelt es sich um ganz junge Bildungen, wahrscheinlich mittelalterlich (vgl. PYRITZ 1972).

\section{Sedimente der Stolzenauer Terrasse}

\subsection{Kiesige Sande mit hohem organischen Anteil}

Die größeren Paläomäander besitzen in der Regel eine Basis aus (kiesigen) Sanden, denen Holzstücke und andere Pflanzenteile in recht großer Zahl beigemengt sind (vgl. Abb. 3, 4). Häufig sind dünne Muddelagen eingeschaltet. Dieser Faziestyp wird insbesondere an den Prall- und Gleithängen recht mächtig. Unter den Gleithängen zieht er bis zu mehreren $100 \mathrm{~m}$ aus der Rinne heraus, bis er zwischen Hochflut-/Auelehmdecken und sterilen Sanden und Kiesen auskeilt. Im zentralen Teil der Rinnen, dort, wo die Rinnensedimente am mächtigsten sind, ist er häufig zerschnitten worden. Die neu entstandenen Rinnen wurden dann mit jüngeren Sedimenten verfüllt. Muddelagen und Holzteile ermöglichen eine pollenanalytische bzw. ${ }^{14} \mathrm{C}$-Datierung dieser Sande. Da Mudden als Stillwassersedimente erst abgelagert werden, wenn die Rinne (zumindest vorübergehend) vom Fluß nicht mehr benutzt wird, ermöglicht die Pollenanalyse der tiefstgelegenen Mudden die Bestimmung eines Mindestzeitpunkts, vor dem die Rinne gebildet wurde. Für alle großen Paläomäander der Stolzenauer Terrasse läßt sich als Mindestalter das Spätglazial angeben (vgl. Tab. 1).

\subsection{Feinklastige Sedimente}

Die feinklastischen Sedimente bestehen aus Mudden und Hochflut-/Auelehmen. Die Geländeansprache 


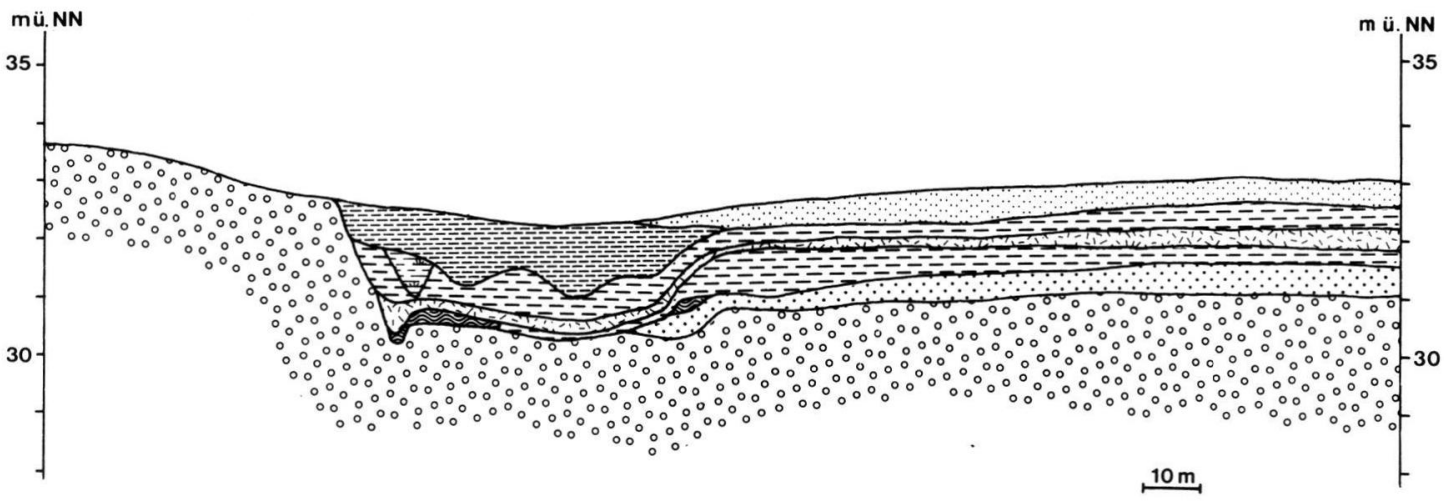

Abb. 3: Profil A-B.

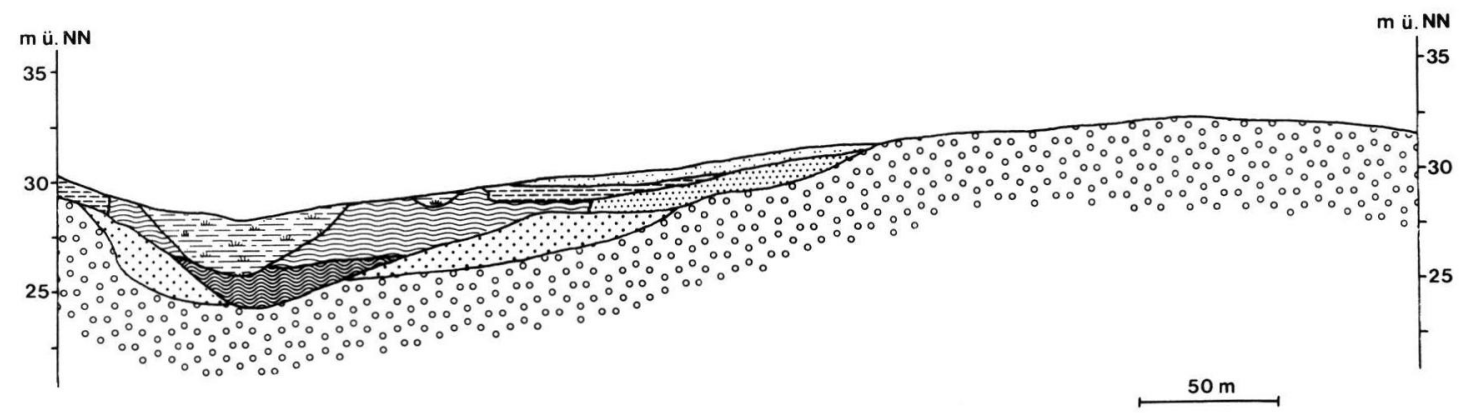

Abb. 4: Profil C-D.

Legende s. Abb. 2

kann anhand von Konsistenz und makroskopischem Eindruck (bei Mudden meist Pflanzenhäcksel deutlich erkennbar) in der Regel eindeutig durchgeführt werden, wobei Übergangstypen vorkommen. Bei den Laboruntersuchungen ergab sich ein derartig eindeutiges Bild nicht. Vielmehr zeigte die Glühverlustanalyse ganz klar, daß der Anteil organischer Substanz allein von der Korngröße des Sediments abhängig ist. Bei vergleichbarer Korngröße unterscheiden sich Mudden und Hochflut-/Auelehme nicht. Insgesamt lag der Gehalt an organischer Substanz in der Trockenmasse selten über $5 \%$. Die feinklastischen Sedimente konnten anhand der Korngrößenanalyse jeweils einem von 6 Korngrößenverteilungstypen (vgl. Kap. 3.2.3) zugeordnet werden, wobei sowohl Mudden als auch Hochflut-/Auelehme bei allen Typen vertreten sind. Die Mudden der Paläomäander sind somit als Sonderform der Hochflut-/Auelehme anzusehen. In den Mudden überwiegt der fluviatile, d. h. mineralische Anteil zu etwa 95\%, während die Stillwassersedimentation mit etwa $5 \%$ organischer Substanz an ihrer Entstehung beteiligt ist. Dennoch erscheint es schon aufgrund der eindeutig möglichen Geländeansprache gerechtfertigt, hier von Mudden zu sprechen, die dann entsprechend als Schluff- und Sandmudden zu klassifizieren sind. Der vergleichbar hohe Anteil organischer Substanz in den Hochflut-1 Auelehmen dürfte auf Humus aus terrestrischen Bodenbildungsprozessen zurückzuführen sein.

\subsubsection{Mudden}

Mudden treten entweder in reiner Form oder (an der Basis) in Wechsellagerung mit kiesigen Sanden auf. Im zentralen Bereich der Rinne, wo die Rinnensedimente am mächtigsten sind, erreichen auch die Mudden ihre größten Mächtigkeiten. Der Bereich größter 
Tab. 1: Fluviatile Dynamik im Spätglazial und Holozän

\begin{tabular}{|c|c|c|c|c|c|c|}
\hline $\begin{array}{l}\text { Jahre } \\
\text { AD }\end{array}$ & \multicolumn{2}{|c|}{$\begin{array}{c}\text { Stratigraphie } \\
\text { n. OVERBECK } 1975\end{array}$} & \multicolumn{2}{|c|}{$\begin{array}{l}\text { Pollenzonen } \\
\text { n. OvERBECK }\end{array}$} & $\begin{array}{l}\text { Kulturperioden } \\
\text { in NWD }\end{array}$ & $\begin{array}{l}\text { Sedimentations- } \\
\text { perioden M-Weser }\end{array}$ \\
\hline \multirow{3}{*}{$1000-$} & \multirow{13}{*}{ 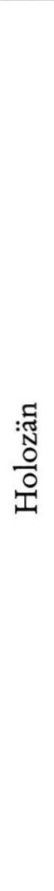 } & \multirow{5}{*}{ Subatlantikum } & \multirow{2}{*}{\multicolumn{2}{|c|}{ XII }} & Neuzeit & \multirow{3}{*}{$\begin{array}{l}\text { Auelehm-Sedimenta- } \\
\text { tion (qh }(2,3) \text { ) } \\
\text { Mudden, selt. Torf }\end{array}$} \\
\hline & & & & & Mittelalter & \\
\hline & & & \multirow{2}{*}{\multicolumn{2}{|c|}{ XI }} & Völk. Wand. Zeit & \\
\hline \multirow[t]{2}{*}{$0-$} & & & & & Röm. Kaiserzeit & \multirow{5}{*}{$\begin{array}{l}\text { mit Bronzezeit } \\
\text { einsetzende } \\
\text { Auelehm-Sedimenta- } \\
\text { tion (qh (1)) } \\
\text { in Rinnen: Torfe } \\
\text { und Mudden, viel } \\
\text { organ. Substanz }\end{array}$} \\
\hline & & & \multicolumn{2}{|c|}{$\mathrm{X}$} & Vorröm. Eisenzeit & \\
\hline \multirow{3}{*}{$\begin{array}{l}-2000- \\
-3000\end{array}$} & & \multirow[b]{2}{*}{ Subboreal } & \multirow{2}{*}{\multicolumn{2}{|c|}{ IX }} & Bronzezeit & \\
\hline & & & & & \multirow[b]{2}{*}{ Jüngere Steinzeit } & \\
\hline & & \multirow{3}{*}{ Atlantikum } & \multirow{2}{*}{\multicolumn{2}{|c|}{ VIII }} & & \\
\hline \multirow{3}{*}{$\begin{array}{l}-5000- \\
-6000-1\end{array}$} & & & & & \multirow{5}{*}{ Mittlere Steinzeit } & \\
\hline & & & & & & \\
\hline & & \multirow{2}{*}{ Boreal } & & & & \\
\hline \multirow{2}{*}{$\begin{array}{l}-7000- \\
-8000-\end{array}$} & & & \multicolumn{2}{|c|}{ VI } & & \\
\hline & & Präboreal & \multicolumn{2}{|c|}{ V } & & \multirow{6}{*}{$\begin{array}{l}\text { in Rinnen: Torfe } \\
\text { und Mudden, rel. } \\
\text { viel organ. Substanz, } \\
\text { kalkhaltig } \\
\text { auf Flächen: } \\
\text { Hochflutlehm } \\
\text { (geringe Ausdehnung) }\end{array}$} \\
\hline \multirow{3}{*}{$\begin{array}{l}-9000- \\
-10000-\end{array}$} & \multirow{5}{*}{ 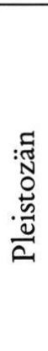 } & Jüng. Parktundra & & & \multirow{5}{*}{ Ältere Steinzeit } & \\
\hline & & Alleröd & & $b$ & & \\
\hline & & Ält. Parktundra & \multirow{2}{*}{ II } & $\mathrm{b}$ & & \\
\hline \multirow{2}{*}{$\begin{array}{l}-11000- \\
-12000-\end{array}$} & & Bölling & & $\mathrm{a}$ & & \\
\hline & & $\begin{array}{l}\text { Baumlose } \\
\text { Tundrenzeit }\end{array}$ & & & & \\
\hline
\end{tabular}

Mächtigkeit der Rinnensedimente liegt in Paläomäandern meist in relativer Nähe zum Prallhang, also dort, wo der Fluß sich am tiefsten eingeschnitten hat. Zum gleichen Ergebnis kam auch KOZARSKI (1983) an der Warthe. Die Mudden keilen zum Gleithang hin aus. Im Gegensatz zu den in Kap. 3.1 beschriebenen kiesigen Sanden verlassen sie aber den eigentlichen Rinnenbereich nicht.

In der Regel konnten die basalen Mudden in den großen Paläomäandern der Stolzenauer Terrasse mit Hilfe der Pollenanalyse in das Spätglazial datiert werden. Jedoch wurde diese spätglaziale Fazies in einigen Fällen während des Holozäns zerschnitten und anschließend mit jüngeren Mudden verfüllt. Einen Anhaltspunkt für das Alter der Mudden in den Paläomäandern kann die Kalkgehaltsbestimmung liefern. In Tiefen bis etwa $2 \mathrm{~m}$ sind die feinklastischen Sedimente der Stolzenauer Terrasse fast vollständig entkalkt (Restkalkgehalte von bis zu $2 \%$ treten auf). Unterhalb dieser Tiefe läßt sich dagegen in zahlreichen Fällen ein Anstieg des Kalkgehaltes auf $5 \%$ und darüber feststellen. Es besteht ein (nicht immer eindeutiger) Zusammenhang zwischen Kalkgehalt und 
Alter des Sediments. Spätglaziale Mudden sind in der Regel unterhalb von etwa $2 \mathrm{~m}$ kalkhaltig, holozäne Mudden dagegen auch in der Tiefe nahezu kalkfrei. Es liegt nahe, für die holozänen Mudden eine primäre Kalkarmut anzunehmen. Als Ursache für den Kalkgehalt der spätglazialen Mudden dürfte in erster Linie ein erhöhter Anteil kalkhaltigen Lösses im Ausgangsmaterial des mineralischen Bestandteils in Frage kommen. Die zunehmende Entkalkung des Lösses im Einzugsgebiet der Weser-Hochwässer könnte dann im Verlauf des Holozäns zu einem primär niedrigen Kalkgehalt im Sediment geführt haben.

\subsubsection{Hochflut-/Auelehm}

Die bisher übliche Unterscheidung zwischen weichselzeitlichem Hochflutlehm und holozänem Auelehm ist in dieser Form nach den vorliegenden Untersuchungen nicht mehr haltbar. Deshalb wird hier der Ausdruck „Hochflut-/Auelehm” für einen Sedimenttypus verwendet, der bei Hochwässern von einem mäandrierenden Fluß außerhalb des Flußbettes (also in der Aue) abgelagert wird. Zur Sedimentation von Hochflut-/Auelehm ist es an der Mittelweser sowohl während des Spätglazials als auch während des Holozäns gekommen, wobei der holozäne Anteil überwiegt. Fast alle größeren und einige der kleineren Paläorinnen der Stolzenauer Terrasse weisen in ihrer unmittelbaren Umgebung (meist auf den tiefergelegenen Gleithang beschränkt) einen Hochflut-/Auelehmstreifen auf (vgl. Abb. 2). Manchmal greift der Hochflut-/Auelehm auch auf die eigentliche Rinne über. Zum höhergelegenen Mäanderkern hin keilt der Hochflut-/ Auelehm aus. Es liegt also nicht, wie in der rezenten Aue, eine geschlossene Hochflut-/Auelehmdecke vor, sondern die Verbreitung ist nur lokal und an die tieferen Lagen gebunden. Verhältnismäßig weite Verbreitung haben die Lehme westlich von Schinna und Anemolter, wo sie früher sogar abgeziegelt wurden. Pollenanalytisch konnten fast alle Lehme der Stolzenauer Terrasse ins Mittelalter datiert werden, was wegen ihrer Lage von $2-3 \mathrm{~m}$ über der rezenten Auelehmdecke der Talaue erstaunen mag. Die Vermutung liegt nahe, daß es sich um ein zeitgleiches Äquivalent des spätmittelalterlichen $\mathrm{qh}(2)$ / S-Auelehms der Weseraue (vgl. LIPPS 1987, 1988) handelt. Dieses schluffig-feinsandige, also verhältnismäßig grobkörnige Sediment ist während der Klimaverschlechterung im 14. und 15. Jahrhundert abgelagert worden. Außerordentliche Starkregen ermöglichten Hochwässer, deren Ausmaß weit über das uns heute bekannte $\mathrm{Maß}$ hinausgegangen sein dürfte. Eine Überschwemmung der Paläorinnen der Stolzenauer Terrasse mit Auelehmablagerung im Spätmittelalter erscheint daher durchaus möglich. Schluffund Tongehalt dieser Lehme sind deutlich höher als im qh(2)/S-Auelehm, was sicherlich auf die Flußferne der Paläorinnen und die verlangsamte Fließgeschwindigkeit der Hochwässer in diesen Bereichen zurückzuführen ist.

Nur in einem Fall gelang eine verhältnismäßig sichere Datierung eines Hochflut-/ Auelehms ins Spätglazial. Einer der großen Paläomäander der Stolzenauer Terrasse (bei Sögeberg) besitzt eine Lehmdecke, in der ein fluviatil umgelagerter Tuff weite Verbreitung besitzt. Der tuffhaltige Horizont erreicht Mächtigkeiten von bis zu mehreren dm und konnte in zahlreichen Bohrungen nachgewiesen werden (vgl. Abb. 3). Es ist der erste Fund dieser Art im Gebiet der Mittelweser. Aufgrund der Mächtigkeit des Horizontes kann es sich nur um Laacher See-Tuff (ca. 9000 v. Chr.) handeln. Der Hochflut-/Auelehm wäre also im Alleröd abgelagert worden. Ein geringmächtiges in situ-Vorkommen des Laacher See-Tuffs konnte noch im Seck-Bruch bei Hannover (DIETZ, GRAHLE \& H. MÜLLER 1958) nachgewiesen werden. Bei dem Vorkommen auf der Stolzenauer Terrasse handelt es sich eindeutig um umgelagertes Material, dem ein hoher Anteil normalen Hochflutsediments beigemischt ist. Dennoch ist der Horizont im Gelände stets eindeutig zu erkennen. Er ist weißlich-grau oder blaßoliv gefärbt und äußerst krümelig. Oben und unten geht er in den Hochflut-/Auelehm über. Demnach enthält das Material vorwiegend Feldspate und Phyllosilikate, daneben Pyroxene. Auch vulkanische Gläser sind enthalten. Die Phyllosilikate dürften zum größten Teil nicht-vulkanischen Ursprungs sein. Aufgrund des hohen Tongehalts ist eine genauere Aussage nicht möglich (schr. Mitt. von RIEZEBOS, 14.11.88). Es muß sich um feinen vulkanischen Staub gehandelt haben, der im Einzugsgebiet der Weser abgelagert und schon kurze Zeit später durch Starkregen abgeschwemmt und im Wesersediment abgelagert worden ist.

\subsubsection{Korngrößenverteilungstypen}

Mudden und Hochflut-/Auelehme der Stolzenauer Terrasse können aufgrund der Zusammensetzung ihrer mineralischen Fraktion jeweils einem von 6 Korngrößenverteilungstypen zugeordnet werden. Typ A zeichnet sich durch einen hohen Mittelsandanteil (um $40 \%$ ) aus. Schluff ist mit rund $15 \%$, Ton mit rund $25 \%$ vertreten. Dieser Typus tritt nur selten und am Rand der Stolzenauer Terrasse auf. Die Sedimente sind durchweg ins Spätglazial datiert. Der hohe Mittelsandanteil ist wahrscheinlich auf Zufuhr von Abschlämmassen von der Niederterrasse zurückzuführen. Typ B ist häufig in den großen Paläomäandern vertreten. Grobschluff herrscht mit $25-45 \%$ deutlich vor, der Tongehalt liegt bei $25 \%$. Die Sedimente 
des Typs B sind ebenfalls recht alt (Spätglazial bis Präboreal).

Aus dem Holozän stammen die Sedimente der Typen $\mathrm{C}$ bis $\mathrm{F}$. Der selten vertretene Typ $\mathrm{C}$ wurde am weserwärtigen Rand der Stolzenauer Terrasse seit dem ausgehenden Boreal sedimentiert. Auffällig ist der hohe Tongehalt (40-50\%). Daneben tritt Mittelsand mit 5-10\% deutlich hervor. Sehr häufig kommt der Typ $\mathrm{D}$ vor. Die Schlufffraktion ist mit $40-50 \%$ vertreten, der Tongehalt liegt bei $20 \%$. Auch hier fällt ein hoher Mittelsandanteil (10-25\%) auf. Es handelt sich durchweg um Sedimente aus dem jüngeren Holozän (Subboreal und Subatlantikum), die meist ebenso wie Typ C an der wesernahen Seite der Stolzenauer Terrasse abgelagert wurden. Seit der römischen Kaiserzeit wurden Sedimente des Typs E am weserfernen Rand der Stolzenauer Terrasse abgelagert. Grobschluff und Feinsand herrschen mit je etwa $30 \%$ vor. Sedimente des Typs E treten relativ selten auf. Häufiger kommt Typ F vor. Es handelt sich um sehr junges Material (jünger als $1400 \mathrm{n}$. Chr.), das wesernah auf der Stolzenauer Terrasse zur Ablagerung kam und sich vorwiegend aus Grobschluff und Ton (je etwa $35 \%$ ) zusammensetzt.

\subsection{Torfe}

In den Rinnen der Stolzenauer Terrasse sind sowohl Anmoorbildungen als auch recht mächtige Niedermoortorfe aus dem Spätglazial und Holozän vertreten. Auch in den muldenförmigen Rinnen des höheren Niveaus sind sie, meist im Gegensatz zu Mudden, hier und da vertreten. Für die Muddenbildung ist ein ganzjährig bestehendes limnisches Milieu erforderlich. Hierfür dürfte in den schmalen, hochgelegenen Rinnen des höheren Niveaus der Grundwasserstand in der Regel nicht ausgereicht haben. Dagegen konnten sich in den tiefergelegenen Bereichen dieser Rinnen, die oft kastenförmig erweitert sind, teilweise bis heute kleine Niedermoore halten. Demzufolge sind die Torfe auf dem höheren Niveau oft sehr jung, teilweise datieren sie aber auch bis ins Spätglazial zurück.

Weite Verbreitung besitzen Niedermoortorfe in den zentralen Bereichen der großen Paläorinnen. Auch hier konnten an den tiefsten und daher feuchtesten Stellen bis in die jüngste Zeit hinein Niedermoore aufwachsen. Zum Gleithang hin keilen die Torfe an der Oberfläche aus oder lösen sich in Torflinsen auf.

In der Regel sind die Niedermoortorfe jünger als die Mudden in der jeweiligen Paläorinne oder dem jeweiligen Rinnenabschnitt. Meist ist der Torf in einer Rinne aufgewachsen, die sich entweder nach vollständiger Verlandung des Altarms erneut eingeschnitten hat oder die bei unvollständiger Verlandung noch nicht mit Mudde verfüllt war. Dies kann nur bei abgesunkenem Wasserstand geschehen sein, verursacht vielleicht teilweise durch die Eintiefung der Weser im Atlantikum (vgl. LIPPS 1987, 1988), die zur morphologischen Ausbildung der Stolzenauer Terrasse geführt hat. Phasen sinkenden und steigenden Grundwasserstandes muß es auf der Stolzenauer Terrasse aber auch zu anderen Zeiten gegeben haben, da es einerseits bereits im Spätglazial zu einer gewissen Torfbildung gekommen ist, andererseits aber die Muddebildung in den Paläorinnen im Mittelalter einen Höhepunkt erreichte. Als Ursachen kommen sowohl überregionale Einflüsse (Klimaschwankungen) als auch regionale Einflüsse (Entfernung vom Vorfluter Weser) in Frage.

\subsection{Abschlämmassen}

Die muldenförmigen Rinnen des höheren Niveaus der Stolzenauer Terrasse sind größtenteils mit Abschlämmassen verfüllt. Es handelt sich um sehr unterschiedliches Material, meist mit hohem Sandanteil, das überwiegend durch mittelalterliche und neuzeitliche Bodenerosionsprozesse in die Rinnen gespült wurde. Die pollenanalytische Datierung von geringmächtigen Muddeschichten im Liegenden der $\mathrm{Ab}$ schlämmassen führte zu dem geringen Alter dieser Sedimente. In den meisten Fällen sind die Sedimente der Rinnen des höheren Niveaus der Stolzenauer Terrasse erstaunlich jung, so daß in einigen Fällen eine spätmittelalterliche Entstehung in Zusammenhang mit den Starkregen des 14. und 15. Jahrhunderts nicht auszuschließen ist. Dies kann jedoch nicht für alle Rinnen auf dem höheren Niveau gelten, da in einigen Fällen spätglaziale bis subboreale Rinnenfüllungen nachgewiesen werden konnten.

\section{Schriftenverzeichnis}

Dietz, C., Grahle, H.-O. \& Müller, H. (1958): Ein spätglaziales Kalkmudde-Vorkommen im Seck-Bruch bei Hannover. - Geol. Jb. 76: 67-102, 9 Abb.; Hannover.

KozARSKI, S. (1983): The Holocene Generation of Paleomeanders in the Warta River Valley, Great Polish Lowlands. - Geol. Jb. A 71: 109-118, 5 Abb.; Hannover.

LIPPS, S. (1987): Abschlußbericht zum Forschungsvorhaben „Fluviatile Dynamik nordwestdeutscher Flüsse im Jungquartär". - DFG-Aktenzeichen $\mathrm{Hi} \mathrm{355/1-2:} 66 \mathrm{~S}$., 5 Abb., 4 Tab., 37 Prof., 2 Kt., mit einem Anhang von H. MÜller: 15 S., 2 Tab.; (unveröff. Ber. NLfB); Hannover. 
LIPPS, S. (1988): Fluviatile Dynamik im Mittelwesertal während des Spätglazials und Holozäns. - Eiszeitalter u. Gegenwart, 38: 78-86, 4 Abb., 1 Tab.; Hannover.

LUTTIG, G. \& MEYER, K.-D. (1980): Erläuterungen zur Geol. Karte von Niedersachsen, 1:25000, Blatt 3320 Liebenau. - 118 S., 9 Abb., 12 Tab., 7 Kt.; Hannover.

MỨleR, H. (1956): Ein Beitrag zur holozänen Emstalentwicklung zwischen Meppen und Dörpen auf Grund von pollenanalytischen Untersuchungen. - Geol. Jb., 71: 491-504, $6 \mathrm{Abb}$.

OVERBECK, F. (1975): Botanisch-geologische Moorkunde. 719 S.; Neumünster.

PYRITZ, E. (1972): Binnendünen und Flugsandebenen im Niedersächsischen Tiefland. - Göttingen Geogr. Abh., 61, 153 S., 27 Abb., 3 Beil.; Göttingen.
Roeschmann, G. (1960): Die Grundwasserböden des Emstales zwischen Rheine und Papenburg und ihre Beziehungen zur Geschichte des Emslaufes. - Geol. Jb., 77: 741-820, 2 Taf., 11 Abb., 6 Tab.; Hannover.

SCHIRMER, W. (1980): Exkursionsführer zum Symposium Franken. Holozäne Talentwicklung - Methoden und Ergebnisse. - 210 S., Düsseldorf.

- (1983): Die Talentwicklung an Main und Regnitz seit dem Hochwürm. - Geol. Jb. A 71: 11-43, 9 Abb.; Hannover.

Manuskript eingegangen am 22. 2. 1989, Nachträge Februar 1990 